\title{
Aprendizagem de gráficos de cinemática por meio da videoanálise associada à metodologia peer instruction
}

\section{Learning kinematics graphics using videoanalysis and peer instruction methodology}

\author{
Mariana de Almeida Jotta Barros*1,2, Vitor Luiz Bastos de Jesus ${ }^{2}$ \\ ${ }^{1}$ Secretaria de Estado de Educação do Rio de Janeiro \\ ${ }^{2}$ Instituto Federal de Educação, Ciência e Tecnologia do Rio de Janeiro
}

\begin{abstract}
Resumo
A interpretação de gráficos pode ser considerada uma habilidade integradora no cotidiano visto que diversas áreas do conhecimento utilizam dados e informações representados na forma de gráficos. Este trabalho apresenta uma sequência didática que utiliza a videoanálise associada à metodologia peer instruction, conhecida em língua portuguesa como aprendizagem por pares (MÜLLER 2017), cujo objetivo é amenizar as dificuldades apresentadas pelos alunos no que tange a interpretação de gráficos em cinemática. A sequência didática foi aplicada em uma escola de ensino médio da rede pública estadual de ensino e organizada em cinco etapas, distribuídas em nove aulas, sendo adaptada ao ensino experimental em escolas desprovidas de laboratório didático. A metodologia foi validada via pré e pós-testes utilizando a versão 4.0 do TUG-K (Test of Understanding Graphs in Kinematics). Os resultados obtidos foram analisados qualitativa e quantitativamente. Os ganhos de Hake são semelhantes aos que Mazur (2015) espera de alunos submetidos a aulas tradicionais. Apesar disso, a proposta deste trabalho permite que os alunos ganhem mais espaço como protagonistas do processo de ensino e aprendizagem, e abre as portas para a experimentação em escolas que não dispõem de laboratórios didáticos.
\end{abstract}

Palavras-chave: Ensino de gráficos de cinemática. Videoanálise. Peer instruction

*mariana_jbarros@yahoo.com.br 


\begin{abstract}
The interpretation of graphs can be considered an integrative skill in everyday life since several areas of knowledge represent data and information as graphs. This paper presents a didactic sequence that uses video analysis associated with peer instruction methodology, which aims to reduce the difficulties presented by the students regarding the interpretation of graphs in kinematics. The didactic sequence was applied in a public state high school system and organized in five stages, distributed in nine classes, being adapted to experimental teaching in schools without a didactic laboratory. The methodology was validated via pre and post tests using the TUG-K version 4.0 (Test of Understanding Graphs in Kinematics). The obtained results were analyzed qualitatively and quantitatively. The Hake's gains are similar to what Mazur (2015) expects from students attending traditional classes. Nevertheless, the proposal of this work allows students to gain more space as protagonists of their teaching and learning process and paves the way for experimentation in schools that cannot afford didactic laboratories.
\end{abstract}

Keywords: Teaching kinematic graphs. Video analysis. Peer instruction

\title{
I. INTRODUÇÃO
}

A interpretação de gráficos é extremamente importante no ensino de física. Os gráficos são tão fundamentais para os físicos, que Beichner (1994) os considera uma segunda linguagem.

Segundo Beichner (1996), a principal confusão que os alunos fazem ao se depararem com gráficos de cinemática está na crença que os gráficos funcionam como uma espécie de fotografia do movimento.

Da Silva (2016) aplicou uma sequência didática utilizando a videoanálise como ferramenta didática para abordar conceitos de gráficos de cinemática em conjunto com a metodologia POE (sigla para Predict, Observe, Explain), em turmas do primeiro ano do ensino médio técnico. A metodologia foi proposta com a intenção de trabalhar com conflitos cognitivos para alcançar resistências profundas nos estudantes, na forma de suas concepções prévias. Contudo, o resultado obtido após o emprego da sequência didática apontou problemas no aprendizado dos gráficos de cinemática.

Este trabalho apresenta uma sequência didática que utiliza a videoanálise associada à metodologia peer instruction, conhecida em língua portuguesa como aprendizagem por pares (MÜLLER 2017), cujo objetivo é amenizar as dificuldades apresentadas pelos alunos no que concerne a leitura de gráficos em cinemática. A sequência didática foi organizada em cinco etapas, distribuídas em nove aulas, sendo adaptada ao ensino experimental em escolas desprovidas de laboratório didático.

Foi utilizado o teste conceitual TUG-K (Test of Understanding Graphics in Kinematics) versão 4.0 como pré-teste e pós-teste para calcular o ganho de Hake e avaliar a sequência didática proposta. A avaliação assimila como o uso da videoanálise de diferentes tipos de movimentos abordados pela metodologia ativa peer instruction podem influenciar na 


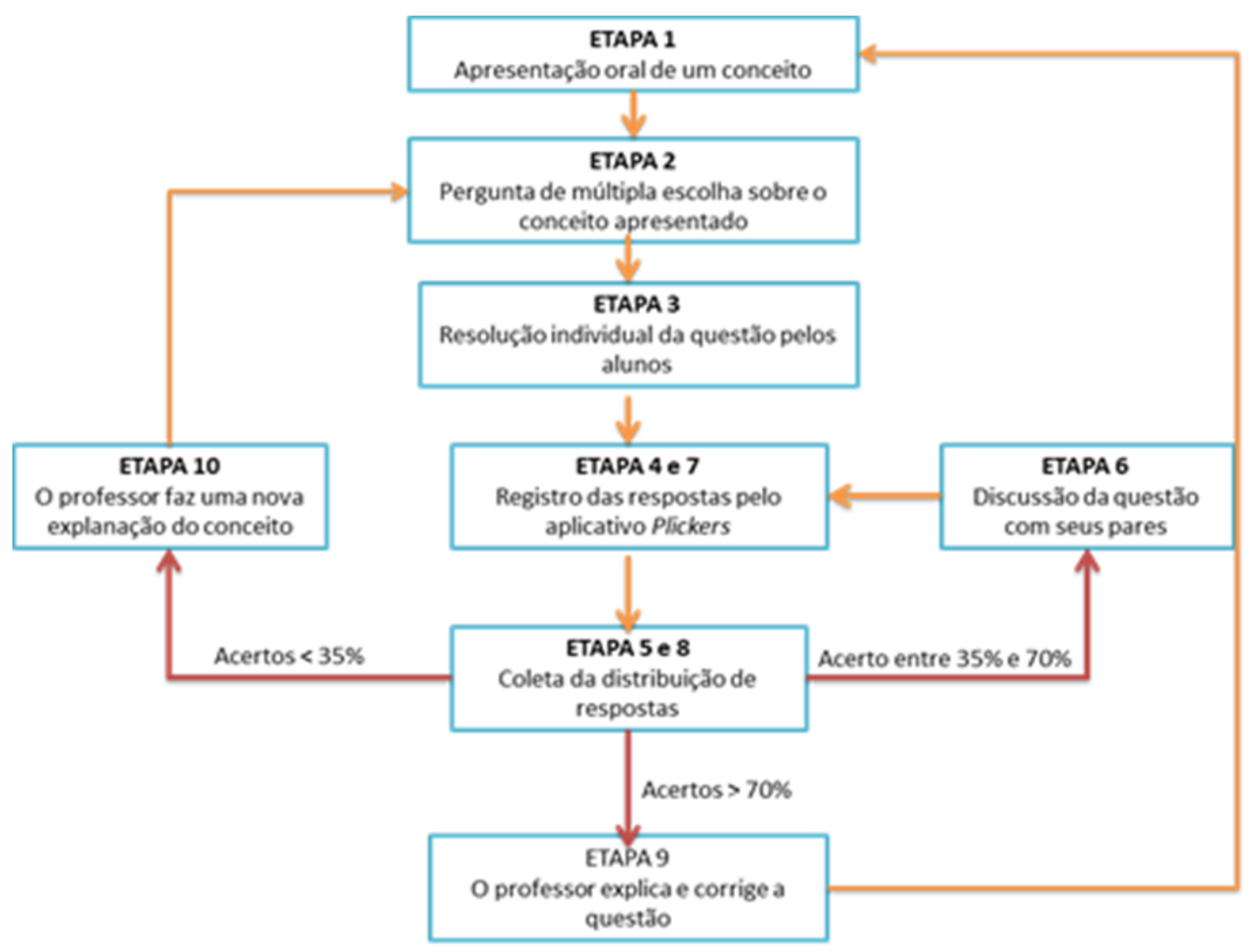

Figura 1: Diagrama de implementação da metodologia peer instruction. Adaptado de ARAUJO e MAZUR (2013).

aprendizagem de conceitos relacionados a interpretação de gráficos de cinemática pelos alunos do ensino médio da rede pública estadual de educação.

As aulas propostas consistem na videoanálise de movimentos de diferentes tipos de fenômenos físicos utilizando o software livre Tracker (BROWN, 2009). Essa ferramenta pode ser considerada uma alternativa de experimentação para escolas que não possuem laboratórios didáticos, mas que tenham disponível em seu espaço físico um laboratório de informática.

O Tracker é um software livre e de código aberto (BEZERRA JUNIOR et al., 2011). Permite realizar análise de vídeos quadro a quadro onde a posição do objeto é observada a cada intervalo de tempo possibilitando a obtenção de sucessivas posições e permitindo o estudo de diversos tipos de movimento a partir de filmes feitos com câmaras digitais ou webcams e computadores comuns.

O método peer instruction (PI) tem foco na aprendizagem conceitual e no desenvolvimento de habilidades sociais e cognitivas (CROUCH; MAZUR, 2001) utilizando atividades que visam à aprendizagem de conceitos teóricos básicos no qual os estudantes explicam tais conceitos a outros estudantes. O método foi desenvolvido na década de 1990 pelo professor de física Eric Mazur da Universidade Harvard. As etapas da metodologia peer instruction estão descritas e ilustradas na Figura 1.

A fim de reportar os problemas encontrados pelos estudantes na interpretação de gráficos de cinemática, Beichner (1994) desenvolveu o teste padronizado TUG-K (Test of 
Understanding of Graphics Kinematics) que pode ser utilizado como ferramenta diagnóstica e como instrumento de avaliação do aprendizado.

O teste conceitual TUG-K foi elaborado inicialmente contendo vinte e uma questões, e cada questão possui um objetivo a ser alcançado pelos alunos. Essa versão sofreu algumas modificações. A justificativa para a elaboração de uma nova versão encontra-se em Zavala et al. (2017).

Zavala et al. (2017) produziram uma nova versão do teste TUG-K, a versão 4.0, contendo vinte e seis questões de múltipla escolha com cinco alternativas cada.

A versão traduzida para ao português do TUG-K v4.0 $0^{1}$ se encontra no portal PhysPort (<https://www.physport.org/assessments/assessment.cfm?I=6\&A=TUGK>). Para ter acesso ao arquivo é necessário fazer cadastro no portal, aceitar os termos de compromisso, que permite gerar uma senha para baixar o teste traduzido.

O propósito para aplicar o TUG-K como pré e pós-testes é verificar a razão entre o aumento absoluto obtido nos acertos e o quanto era possível alcançar no teste, razão conhecida como ganho de Hake (HAKE, 1998).

O ganho normalizado de Hake é calculado a partir da seguinte expressão:

$$
g=\frac{\% \text { pos }-\% \text { pre }}{100-\% \text { pre }}
$$

Sendo: g: ganho de Hake, \%pós: percentual de acertos no pós-teste e \%pré: percentual de acertos no pré-teste,

\section{Metodologia}

Com a finalidade de aperfeiçoar a leitura, compreensão e interpretação de gráficos de cinemática dos estudantes do ensino médio, a metodologia deste trabalho foi organizada em cinco etapas, distribuídas em nove aulas. Cada aula é composta de dois tempos de 50 minutos.

A pesquisa foi realizada em quatro turmas de primeiro ano do ensino médio da rede pública estadual de ensino do RJ, com cerca de 40 estudantes por turma. As etapas da sequência didática são apresentadas na Tabela 1.

A primeira etapa consiste em uma aula inaugural e restringe-se a um encontro com a turma, em que é explicada a importância da leitura de gráficos, via questionamentos levantados pelo professor O que é e qual é a função da mecânica clássica?, Qual a importância da leitura de gráficos?, ou Em quais momentos a interpretação de um gráfico se fez presente no seu cotidiano?. Também são apresentadas a metodologia e a videoanálise mediada pela metodologia PI, que será empregada nas próximas aulas.

A apresentação da metodologia PI adotada na aula inaugural tem como objetivo adaptar os alunos às etapas da sequência didática tendo em vista que serão trabalhados novos elementos à realidade do aluno como, por exemplo, a videoanálise e o aplicativo Plickers

\footnotetext{
${ }^{1}$ Tradução do teste feita pelos autores deste artigo e pelo Professor Daniel Guilherme Gomes Sasaki (CEFET-RJ).
} 
Tabela 1: Etapas da sequência didática.

\begin{tabular}{|l|l|l|l|}
\hline Etapa & \multicolumn{1}{|c|}{ Atividade } & \multicolumn{1}{|c|}{ Carga horária } & Instrumentos utilizados \\
\hline 1o & Aula inaugural. & 1h e $40 \mathrm{~min}$ & $\begin{array}{l}\text { Lista de exercícios } \\
\text { com tomada de res- } \\
\text { postas pelo aplicativo } \\
\text { Plickers. }\end{array}$ \\
\hline 20 & $\begin{array}{l}\text { Aplicação do } \\
\text { pré-teste. }\end{array}$ & $1 \mathrm{~h}$ e $40 \mathrm{~min}$ & $\begin{array}{l}\text { Teste conceitual TUG- } \\
\text { K v4.0. }\end{array}$ \\
\hline 30 & $\begin{array}{l}\text { Ambientação ao } \\
\text { laboratório de } \\
\text { informática e ao } \\
\text { software Tracker. }\end{array}$ & $\begin{array}{l}\text { Apresentação do Ma- } \\
\text { nual do software Trac- } \\
\text { ker para estudantes } \\
\text { e videoanálise do ví- } \\
\text { deo Carros na Ave- } \\
\text { nida Brasil. }\end{array}$ \\
\hline 40 & $\begin{array}{l}\text { Aulas combi- } \\
\text { nando video- } \\
\text { análise com a a } \\
\text { metodologia PI. }\end{array}$ & $\begin{array}{l}\text { Videoanálise de di- } \\
\text { ferentes movimentos; } \\
\text { roteiros sobre MRU e } \\
\text { MRUV; metodologia } \\
\text { PI; uso do aplicativo } \\
\text { Plickers. }\end{array}$ \\
\hline 50 & $\begin{array}{l}\text { Aplicação do } \\
\text { pós-teste. }\end{array}$ & 1h e 40 min & $\begin{array}{l}\text { Teste conceitual TUG- } \\
\text { K v4.0. }\end{array}$ \\
\hline
\end{tabular}

(ferramenta para registrar e armazenar as respostas) ${ }^{2}$.

A segunda etapa compreende a aplicação do pré-teste, TUG-K v4.0, que apesar de ter sido desenvolvido para alunos universitários, no presente trabalho foi aplicado em turmas do primeiro ano do ensino médio. A aplicação do pré-teste visa analisar os conhecimentos prévios dos alunos sobre gráficos relacionados à cinemática. A partir dos resultados obtidos no pré-teste foram desenvolvidos vídeos para as atividades videoanálise combinadas com a metodologia PI.

Na terceira, etapa os alunos são levados ao laboratório de informática da escola onde se apresenta a videoanálise e o software livre Tracker (SLT) e a técnica de videoanálise. É ensinado o passo a passo para o uso do SLT utilizando o manual fornecido pelo professor aos alunos. O manual para estudantes encontra-se no Apêndice A e foi desenvolvido pelos autores deste trabalho. É utilizada apenas uma aula para a realização dessa etapa. Os objetivos dessa aula são explicar o que compreende a videoanálise, ambientar os alunos ao laboratório de informática, apresentar o SLT, seu manual e os comandos fundamentais para a realização da videoanálise.

A quarta etapa consiste em um conjunto de 6 aulas. Nela são combinadas atividades de videoanálise à metodologia PI. Seu objetivo é abordar conceitos de cinemática dos movimentos retilíneos uniforme e uniformemente variável, aprimorar a leitura e interpretação de gráficos de cinemática.

Nessa etapa foram desenvolvidas três atividades que fazem uso de tal combinação

\footnotetext{
${ }^{2}$ Pode-se baixar a ferramenta Plickers pelo site https://www.plickers.com/.
} 
(videoanálise e a metodologia PI). Para cada atividade será utilizada uma aula. A primeira atividade trata do movimento retilíneo uniforme, cujo vídeo é a filmagem do movimento de carros em uma via expressa. A segunda atividade trata da videoanálise da queda livre de uma bola de tênis. A terceira trata da videoanálise do experimento lata que vai e volta. Tanto a segunda quanto a terceira atividades abordam conceitos sobre o movimento uniformemente variado.

Os roteiros das atividades são apresentados em duas partes. Na primeira parte são apresentados itens essenciais para iniciar e realizar a videoanálise do movimento a ser estudado. A segunda parte apresenta questões de múltipla escolha sobre conceitos de gráficos de cinemática a cerca do movimento em estudo. Nessa parte, os alunos responderam conforme a metodologia PI propõe e suas respostas foram registradas pelo professor através do aplicativo Plickers.

A execução da atividade conta primeiramente com a realização da videoanálise do movimento pelos alunos, organizados preferencialmente em duplas, que fazem uso das informações presentes na primeira parte do roteiro.

Após a realização da videoanálise, é apresentada a segunda parte do roteiro, sendo os alunos guiados pelo professor a responderem uma questão por vez.

A abordagem de cada questão é feita da seguinte maneira, primeiramente o professor apresenta o conceito acerca da questão que está sendo trabalhada e, em seguida, os alunos fazem a leitura da questão respondendo conforme as etapas da metodologia PI. A resposta de cada aluno é captada e armazenada pelo aplicativo Plickers. Durante a resolução da questão, o aluno pode utilizar a videoanálise como ferramenta de suporte e consulta de dados.

Efetuadas as etapas da metodologia, se a turma atinge o percentual de acertos necessários de aproximadamente $70 \%$, segue-se para a próxima questão, onde todo o processo para a resolução da questão recomeça.

O laboratório de informática no qual foi feito a videoanálise permite que os alunos se agrupem por computador geralmente em duplas ou, em alguns casos, em trios dependendo do número de alunos por turma. Em caso de dúvidas para a realização da videoanálise o aluno poderia consultar o Manual para estudantes e também o professor.

A quinta etapa consiste na aplicação do pós-teste, que trata da reaplicação do teste conceitual TUG-K versão 4.0. É importante que os alunos não tenham acesso à resolução das questões e novamente devem fazer o teste anonimamente.

A seguir serão descritas as atividades desenvolvidas na quarta etapa, que trata de aulas combinando videoanálise com a metodologia PI.

\section{Primeira atividade: movimento retilíneo uniforme (MRU)}

A primeira atividade visa trabalhar conceitos de movimento retilíneo uniforme e para tal foram necessárias 2 aulas (3h e 20min). O vídeo escolhido para videoanálise é uma filmagem de automóveis deslocando-se ao longo da Avenida Brasil, filmado e elaborado pela autora do presente trabalho ${ }^{3}$. Na filmagem os automóveis deslocam-se com velocidade

${ }^{3} \mathrm{O}$ vídeo encontra-se disponível para download em <https://drive.google.com/drive/folders/ 1DmZXcbn6p1iljYDFWzSzDJZ0U_FOwFlk> e para visualização em <https://youtu.be/jBGO157AbpU>. 
constante ao longo da pista. Isto ocorre porque é feito um recorte do movimento, ou seja, é analisado um pequeno trecho do movimento em que a velocidade é praticamente constante (Figura 2).

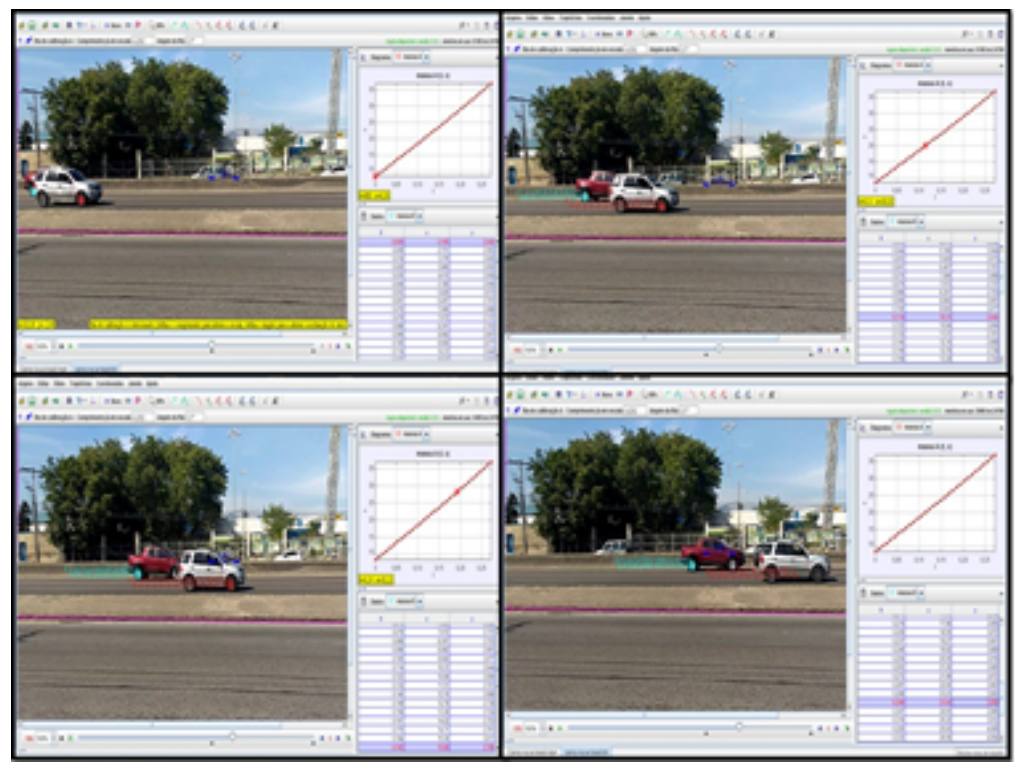

Figura 2: : Imagens da tela do software Tracker durante a videoanálise do movimento dos carros na Avenida Brasil.

A atividade foi desenvolvida com o objetivo de abranger os seguintes conceitos: apresentar as características do movimento uniforme tais como corpos com velocidade constante percorrem distâncias iguais para intervalos de tempos iguais; a velocidade nula não caracteriza uma velocidade constante; compreensão, leitura e análise de gráficos; determinar a velocidade do corpo utilizando do gráfico de posição versus tempo; determinar o deslocamento do móvel utilizando o gráfico de velocidade versus tempo.

No Material Suplementar, seção B, encontra-se o roteiro elaborado para a atividade de movimento retilíneo uniforme.

\section{Segunda atividade: movimento retilíneo uniformemente variável (MRUV)}

Nessa atividade foi utilizada a filmagem feita por De Jesus (2014) de uma bola de tênis em queda livre ${ }^{4}$. A bola é abandonada de uma determinada altura e a videoanálise é feita até o instante em que a bola de tênis toca o solo (Figura 3). A atividade foi iniciada com uma aula expositiva sobre conceitos do movimento retilíneo uniformemente variado e aos gráficos relacionados a este movimento.

No segundo momento foi introduzido o roteiro como as informações necessárias para que o aluno possa fazer a videoanálise do movimento. O roteiro também contém questões elaboradas com o objetivo de facilitar a compreensão dos conceitos básicos sobre MRUV e a aprendizagem da interpretação dos gráficos relacionados ao movimento.

\footnotetext{
${ }^{4} \mathrm{O}$ vídeo encontra-se disponível para download em <https://www.dropbox.com/home/V\%C3\%ADdeos\% 20para\%20videoan \%C3\%A1lise \%20de\%20gr\%C3\%A1ficos\%20de \%20cinem\%C3\%A1tica?preview=Cap+3+-+ Queda+da+bola+de+tenis.mp4>.
} 


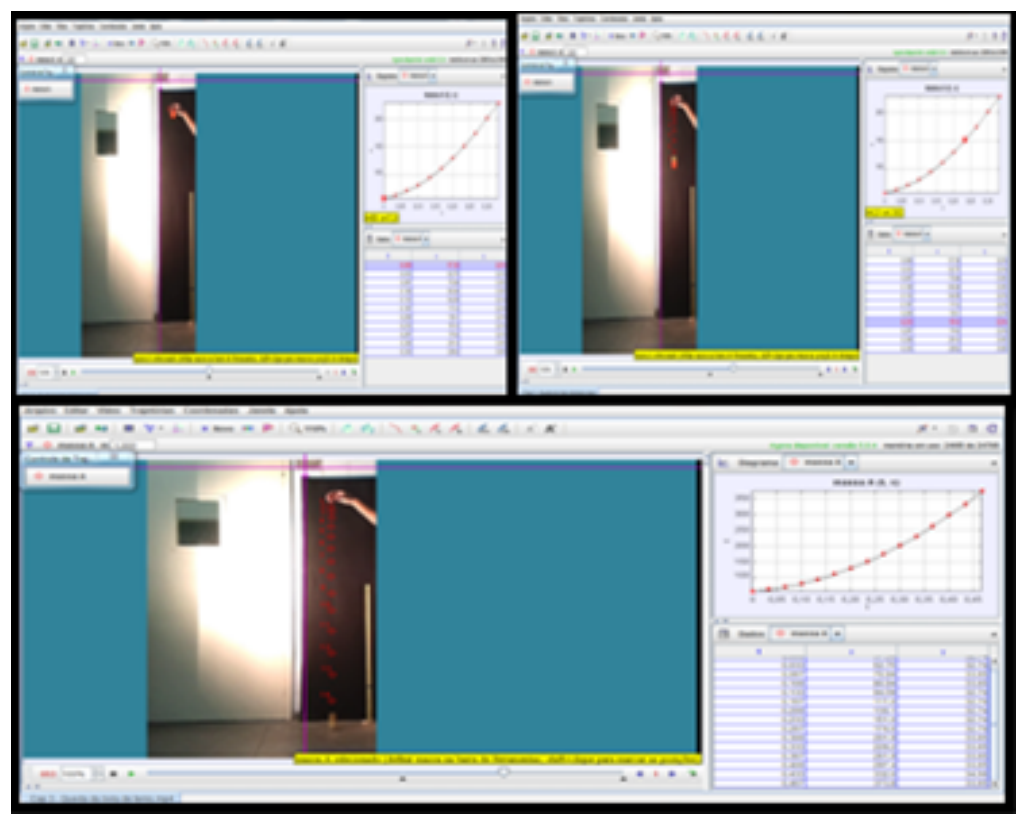

Figura 3: Imagens da tela do software Tracker durante a videoanálise da queda livre de uma bola de tênis, adaptado de De Jesus (2014).

O objetivo da atividade de MRUV consiste em apresentar as características desse movimento, aprimorar a leitura e análise de gráficos, dentre os quais destacam-se, o gráfico de posição versus tempo afim de determinar o sentido do deslocamento, o gráfico de velocidade versus tempo para determinar a aceleração e o gráfico de aceleração versus tempo para determinar a velocidade do corpo, além de medir o valor da aceleração da gravidade. A saber, o primeiro roteiro sobre MRUV encontra-se no Material Suplementar, seção C.

\section{Terceira atividade: experimento da lata que vai e volta}

A terceira atividade é representada pela filmagem do experimento da lata que vai e volta $^{5}$. Os materiais para a construção do experimento são uma lata de metal, dois palitos de dente, um elástico, fita adesiva e quatro pilhas pequenas. A construção do experimento começa na junção das pilhas com fita adesiva, em seguida prende-se o elástico ao conjunto de pilhas também com auxílio da fita adesiva, tudo posto dentro da lata onde o elástico é esticado até passar pelos respectivos furos em cada extremidade da lata, sendo fixados com os palitos em suas respectivas partes externas. O movimento da lata é mostrado na Figura 4.

A filmagem deste experimento, realizada pelos autores deste trabalho, mostra a lata sendo colocada para rolar ao longo de uma superfície plana seguindo para a direita do vídeo até parar completamente e mudar o sentido do movimento para a esquerda. Esse experimento pode ser considerado similar ao movimento de um corpo lançado verticalmente. O objetivo do experimento consiste em reforçar conceitos sobre a influência da aceleração sobre a velocidade, facilitar e fixar a leitura de gráficos de posição versus tempo, velocidade

\footnotetext{
${ }^{5}$ É possível baixar o vídeo a partir do endereço <https://drive.google.com/drive/folders/ 1DmZXcbn6p1iljYDFWzSzDJZ0U_FOwFlk> e a visualização do vídeo encontra-se em <https://youtu.be/ _zSPwu2IXGY>.
} 


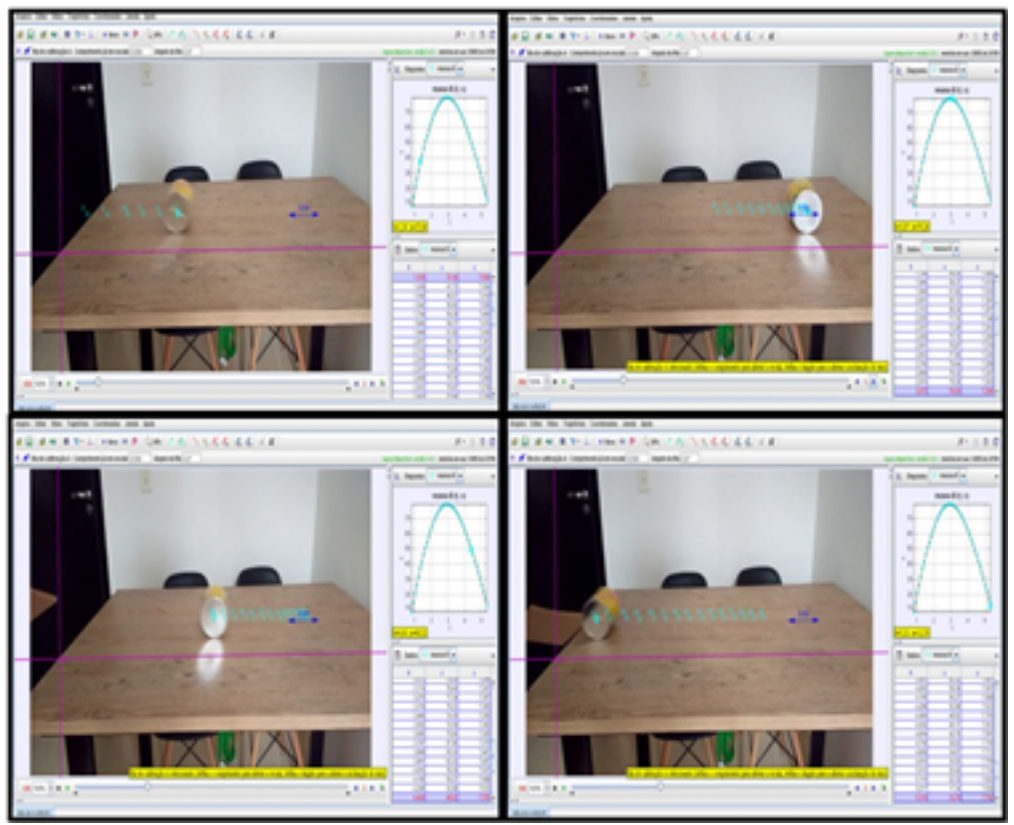

Figura 4: Imagens da tela do software Tracker durante a videoanálise do movimento da lata que vai e volta.

versus tempo e aceleração versus tempo. Por se tratar de um experimento diferente, porém com um tipo de movimento muito presente no dia a dia espera-se que ao final da atividade o aluno seja capaz de identificar e diferenciar um corpo em movimento retilíneo uniforme de um corpo em movimento uniformemente variado. $\mathrm{O}$ roteiro que orienta a terceira atividade encontra-se no Material Suplementar, seção D.

Durante a realização das questões referentes às atividades de videoanálise, presentes nos roteiros, pretende-se incentivar os alunos a participarem e a interagirem entre si, como propõe a metodologia ativa PI.

\section{Resultados E discussões}

No ganho normalizado de Hake, dado pela equação (1), os valores encontrados entre o intervalo de 0 a 1 equivalem a uma porcentagem de $0 \%$ e $100 \%$ de possível ganho na aprendizagem. Quanto mais elevado o valor, maior será o grau de aprendizagem do grupo. Os valores de $\mathrm{g}$ inferiores ou igual a 0 representam que não houve ganho de aprendizagem.

Mazur (2015) salienta que, em turmas que adotam a metodologia PI, o valor médio do ganho deve ser superior a 0,36, enquanto que, para turmas expostas ao método tradicional, o valor médio do ganho deve estar entre 0,1 e 0,2 .

Os dados obtidos no pré- e pós-teste estão apresentados nas tabelas 2 e 3, enquanto que os cálculos do ganho de Hake para as turmas A, B, C e D submetidas à metodologia proposta pelo presente trabalho estão apresentados na tabela 4 . 
Tabela 2: Resultados obtidos no pré-teste.

\begin{tabular}{|c|c|c|c|c|}
\hline \multicolumn{5}{|c|}{ Pré-teste } \\
\hline Turma & Alunos & Total de questões & Total de acertos & Porcentagem de acertos(\%) \\
\hline A & 39 & $39 \times 26=1014$ & 134 & 13,2 \\
\hline B & 29 & $29 \times 26=754$ & 107 & 14,2 \\
\hline C & 33 & $33 \times 26=858$ & 75 & 8,7 \\
\hline D & 36 & $36 \times 26=936$ & 74 & 7,9 \\
\hline
\end{tabular}

Tabela 3: Resultados obtidos no pós-teste.

\begin{tabular}{|c|c|c|c|c|}
\hline \multicolumn{5}{|c|}{ Pós-teste } \\
\hline Turma & Alunos & Total de questões & Total de acertos & Porcentagem de acertos(\%) \\
\hline A & 35 & $35 \times 26=910$ & 181 & 19,9 \\
\hline B & 27 & $27 \times 26=702$ & 113 & 16,9 \\
\hline C & 30 & $30 \times 26=780$ & 135 & 17,3 \\
\hline D & 30 & $30 \times 26=780$ & 116 & 14,9 \\
\hline
\end{tabular}

Tabela 4: Cálculo do ganho de Hake.

\begin{tabular}{|c|c|}
\hline Turma & Ganho de Hake \\
\hline A & 0,08 \\
\hline B & 0,03 \\
\hline C & 0,09 \\
\hline D & 0,08 \\
\hline
\end{tabular}

Os valores do ganho de Hake para as turmas A, B, C e D são positivos e pertencem ao intervalo de 0 a 0,10. Mesmo que esses valores sejam pequenos, eles indicam algum ganho de aprendizagem. Os valores alcançados pelas turmas que fizeram uso da videoanálise em conjunto com a metodologia PI estão dentro da faixa esperada de ganho de Hake que Mazur (2015) considera como metodologia tradicional, isto é, valores entre 0,10 e 0,20.

Os resultados obtidos nos pré e pós-testes pelas turmas A, B, C e D serão analisados estatisticamente utilizando o teste de significância para igualdade entre as médias.

O teste de significância para igualdade entre as médias resumidamente consiste em testar e formular hipóteses através de um espaço amostral indicado e permitir a aceitação ou rejeição da hipótese formulada com base nos resultados de uma amostra. Para tal é necessário enunciar as hipóteses de nulidade, hipótese a ser testada (H0), formular a hipótese alternativa (H1), fixar o limite do erro $(\alpha)$ e com auxílio da tabela de distribuição normal determinar as regiões crítica (R.C.) e de aceitação (R.A.) para H0 (MORETTIN; BUSSAB, 2010).

Para delimitar as regiões R.A. e R.C. é necessário calcular o valor da variável do teste (Zcal) e assim, concluir pela aceitação ou rejeição de H0 (MORETTIN; BUSSAB, 2010).

$$
\text { Zcal }=\frac{\bar{x}_{1}-\bar{x}_{2}}{\sqrt{\frac{\sigma_{1}^{2}}{N 1}+\frac{\sigma_{2}^{2}}{N 2}}}
$$


Tabela 5: Médias e desvios quadráticos médios obtidos no pré- e pós-teste.

\begin{tabular}{|c|c|c|c|c|c|c|c|c|c|}
\hline \multicolumn{5}{|c|}{ Análise pré-teste } & \multicolumn{5}{c|}{ Análise pós-teste } \\
\hline Turma & $\mathrm{A}$ & $\mathrm{B}$ & $\mathrm{C}$ & $\mathrm{D}$ & & $\mathrm{A}$ & $\mathrm{B}$ & $\mathrm{C}$ & $\mathrm{D}$ \\
\hline $\bar{x}_{1}$ & 1,32 & 1,42 & 0,87 & 0,79 & $\bar{x}_{2}$ & 1,99 & 1,61 & 1,73 & 1,49 \\
\hline$\sigma_{1}$ & 0,96 & 1,05 & 0,59 & 0,70 & $\sigma_{2}$ & 1,05 & 0,84 & 1,07 & 0,74 \\
\hline$N_{1}$ & 39 & 29 & 33 & 36 & $N_{2}$ & 35 & 27 & 30 & 30 \\
\hline
\end{tabular}

Tabela 6: Teste de significância entre as médias para o nível de confiança de 1\%.

\begin{tabular}{|c|c|c|c|c|}
\hline \multicolumn{5}{|c|}{ Teste de significância para igualdade entre as médias } \\
\hline Turma & Nível de confiança & $\mathbf{Z}$ & Zcal & H0 \\
\hline A & $1 \%$ & 2,57 & $-2,85$ & Rejeitado \\
\hline B & $1 \%$ & 2,57 & -0.75 & Aceito \\
\hline C & $1 \%$ & 2,57 & -3.88 & Rejeitado \\
\hline D & $1 \%$ & 2,57 & $-3,92$ & Rejeitado \\
\hline
\end{tabular}

Iremos admitir a hipótese alternativa, H1, como sendo a diferença entre as médias nos testes conceituais, pré-teste $\left(\bar{x}_{1}\right)$ e pós-teste $\left(\bar{x}_{2}\right)$, ou seja, $\bar{x}_{1} \neq \bar{x}_{2}$. Desse modo, para H0 iremos admitir que as médias obtidas nos testes conceituais sejam iguais $\left(\bar{x}_{1}=\bar{x}_{2}\right)$.

Por fim, aceitação ou rejeição a hipótese H0, é feita na seguinte afirmação:

$$
\begin{gathered}
\text { Se }-Z \leq Z c a l \leq Z \text {, aceita-se } H 0 \\
\text { Se } Z c a l<-Z \text { ou } Z c a l>Z \text {, rejeita-se } H 0
\end{gathered}
$$

A comparação entre as médias precisa estar na região R.A., pois, quanto mais afastado da região central do espaço amostral, maior será a possibilidade de as médias no pré-teste e no pós-teste serem estatisticamente diferentes. Visto que os valores entre as médias obtidas no pré-teste e no pós-teste serem muito próximos, a execução do teste de significância tem o desígnio de expressar a validade dos testes conceituais.

Se as médias entre os testes forem estatisticamente iguais, aceita-se a hipótese H0. Para esse trabalho significa que os resultados dos testes são inconclusivos.

Nesse trabalho é imperativo que as médias no pré-teste e no pós-teste sejam diferentes, assim é possível verificar se houve ganho ou perda de aprendizagem. Portanto, admitiremos a rejeição da hipótese $\mathrm{H} 0$ como verdade.

Na Tabela 5 são apresentados os dados obtidos através da aplicação dos pré e pós-testes.

Para uma melhor compreensão da faixa de confiança para as médias obtidas nos pré e pós-testes, apresentamos na tabela 6 o teste de significância para igualdade entre as médias para o nível de confiança de $1 \%$.

A análise estatística feita pelo teste de significância para igualdade entre as médias indica que para as turmas A, C e D, as médias obtidas nos pré e pós-testes estão na região de aceitação em uma distribuição normal de 1\%, as médias comparadas podem ser consideradas diferentes.

Esse resultado nos mostra que mesmo que a média do pré-teste pareça ser numericamente próxima à média do pós-teste, pelo teste de significância para igualdade entre as médias, 
Tabela 7: Comparação entre as médias obtidas no pré-teste e no pós-teste dos alunos do IFF Cambuci e das médias obtidas pelos alunos no presente trabalho.

\begin{tabular}{|l|l|l|l|l|l|l|l|l|l|l|}
\hline Turma & \multicolumn{2}{|l|}{ IFF Cambuci } & \multicolumn{2}{|c|}{ A } & \multicolumn{2}{|c|}{ B } & \multicolumn{2}{c|}{ C } & \multicolumn{2}{c|}{ D } \\
\hline TUG-K & $\begin{array}{l}\text { Pré- } \\
\text { teste }\end{array}$ & $\begin{array}{l}\text { Pós- } \\
\text { teste }\end{array}$ & $\begin{array}{l}\text { Pré- } \\
\text { teste }\end{array}$ & $\begin{array}{l}\text { Pós- } \\
\text { teste }\end{array}$ & $\begin{array}{l}\text { Pré- } \\
\text { teste }\end{array}$ & $\begin{array}{l}\text { Pós- } \\
\text { teste }\end{array}$ & $\begin{array}{l}\text { Pré- } \\
\text { teste }\end{array}$ & $\begin{array}{l}\text { Pós- } \\
\text { teste }\end{array}$ & $\begin{array}{l}\text { Pré- } \\
\text { teste }\end{array}$ & $\begin{array}{l}\text { Pós- } \\
\text { teste }\end{array}$ \\
\hline $\begin{array}{l}\text { Alunos } \\
\text { Presentes }\end{array}$ & 74 & 64 & 39 & 35 & 29 & 27 & 33 & 30 & 36 & 30 \\
\hline $\begin{array}{l}\% \text { de acer- } \\
\text { tos }\end{array}$ & 12,8 & 18,9 & 13,21 & 19,89 & 14,19 & 12,42 & 8,74 & 14,84 & 7,91 & 12,75 \\
\hline $\begin{array}{l}\text { Média de } \\
\text { acertos }\end{array}$ & 2,7 & 4,0 & 3,44 & 5,17 & 3,72 & 4,18 & 2,27 & 4,50 & 2,06 & 3,87 \\
\hline $\begin{array}{l}\text { Ganho de } \\
\text { Hake }\end{array}$ & \multicolumn{2}{|c|}{0,07} & \multicolumn{2}{|c|}{0,08} & \multicolumn{2}{|c|}{0,02} & & 0,07 & \multicolumn{2}{|c|}{0,05} \\
\hline
\end{tabular}

elas são estatisticamente diferentes entre si.

Já a turma B, a hipótese de nulidade foi aceita, o que demonstra que as médias estão fora da região de aceitação num limite de erro de $2,5 \%$ e dentro da região crítica. A aceitação da hipótese aponta para igualdade entre as médias para um nível de confiança de 95\% e, portanto, para este intervalo as médias são estatisticamente iguais. Sendo assim, não é possível fazer qualquer afirmação a respeito dessa turma já que a média obtida por essa turma no pré-teste é estatisticamente igual à média no pós-teste.

Em Da Silva (2016) encontramos resultados análogos aos obtidos neste trabalho conforme mostra a Tabela 7. Em sua dissertação o autor utilizou a videoanálise como ferramenta pra o ensino de gráficos de cinemática mediado pela metodologia $\mathrm{POE}$, para alunos do primeiro ano do ensino médio localizado no IFF (Instituto Federal Fluminense) da cidade de Cambuci, pertencente a uma zona agrícola.

Apesar de ter utilizado em sua análise a versão anterior do TUG-K, versão v.2.6, a mesma versão utilizada em Agrello e Garg (1999), a comparação entre Da Silva (2016) com o presente trabalho foi feita com os resultados obtidos pelos alunos no ganho de Hake e nas médias de acerto geral no pré-teste e pós-teste.

O ganho de Hake apresentado na turma do IFF de Cambuci é próximo aos ganhos obtidos nas turmas A, C e D. Inclusive, as médias de acerto geral obtidas nos pré e póstestes, das turmas do IFF Cambuci são muito próximas às médias encontradas nos pré e pós-testes das turmas A, B, C e D.

Assim como os alunos do IFF de Cambuci, os alunos que fizeram parte da metodologia proposta no presente trabalho apresentaram um pequeno ganho de Hake no teste conceitual TUG-K.

\section{Considerações Finais}

Atualmente estamos vivenciando uma quebra de paradigma no ensino de física no qual o método tradicional de ensino, engajado na memorização e matematização dos conceitos, embora muito utilizado nas escolas, vem perdendo espaço para metodologias que visem o 
aluno como protagonista do processo de aprendizagem e busque situações-problemas mais próximas da sua realidade.

A análise quantitativa dos resultados alcançados no presente trabalho mostra que as turmas que participaram da metodologia proposta apresentaram um ganho de Hake entre 0 e 0,10. Segundo Mazur (2015), essa faixa corresponde a um ganho semelhante a uma aula tradicional ao passo que as turmas que adotam a metodologia PI o valor médio do ganho deve ser superior a 0,36 .

Vale ressaltar o ganho de Hake $(\mathrm{g}=0,02)$ apresentado pela turma $\mathrm{B}$, aquela que apresentou o menor desempenho após a aplicação da sequência didática proposta. $\mathrm{O}$ teste de significância mostra que a média obtida pelo pré-teste é estatisticamente igual a média obtida no pós-teste e, portanto, o ganho obtido por essa turma é considerado inconclusivo.

Ainda que os ganhos de Hake apresentados pelas demais turmas (A, C e D) sejam menores do que se espera de turmas que participam de aulas tradicionais, os valores indicam, ainda que pouco, ganhos de aprendizagem.

Corroborando com valores dos ganhos apresentados pelas turmas submetidas à metodologia proposta no presente trabalho encontra-se a pesquisa desenvolvida por Da Silva (2016) na qual os valores obtidos pelo teste conceitual, TUG-K, apontaram pequenos ganhos de aprendizagem de gráficos de cinemática dos alunos de ensino médio após utilizar uma sequência didática que trabalhou a videoanálise de diferentes tipos de movimentos combinada com a metodologia ativa POE.

Em uma reflexão mais profunda, Da Silva (2016) destacou que muitos alunos persistiram com uma série de confusões na interpretação de gráficos mesmo após a aplicação da sequencia didática. Apesar de as sequências didáticas propostas neste trabalho e por Da Silva (2016) utilizarem videoanálise, mas metodologias diferentes, P.I. e POE, respectivamente, os ganhos de Hake obtidos a partir do teste conceitual TUG-K, ainda que em versões diferentes, estão dentro de uma faixa que corresponderia a aulas tradicionais, como elucida Mazur (2015). Entretanto, é possível encontrar na literatura trabalhos que apontam a videoanálise e a metodologia ativa, peer instruction, como instrumentos motivadores e facilitadores do processo ensino-aprendizagem.

De maneira geral, a sequência didática proposta pode se tornar uma ferramenta para facilitar a aprendizagem dos alunos ao que tange a compreensão de gráficos de cinemática. A proposta permite que os alunos ganhem mais espaço como protagonistas do processo de ensino e aprendizagem possibilitam a experimentação em ambientes desprovidos de laboratórios didáticos e a análise de vídeos através da videoanálise corrobora como item motivador, uma vez que, muitos alunos não possuem acesso a computadores no seu cotidiano.

Outro fator importante é que a transição de uma metodologia tradicional para uma metodologia mais ativa envolve muitos processos e desafios. Dentre eles o maior desafio seja levar o aluno a compreenderem seu protagonismo no processo de aprendizagem. Muitos ainda encontram-se presos a reproduzir conhecimentos passados pelo professor, em vista disso é imperativo leva-los vencer a inércia da reprodução para que sejam capazes de pensar por si próprio ao refletir sobre situações-problema. 


\section{AgRadecimentos}

Os autores agradecem aos professores Alexandre Lopes de Oliveira e Daniel Guilherme Gomes Sasaki por suas contribuições nas discussões dos resultados obtidos nesta pesquisa, pela minuciosa leitura e revisão do manuscrito.

\section{REFERÊNCIAS}

ARAUJO, I. S.; MAZUR, E. Caderno Brasileiro de Ensino de Física, Florianópolis, v.30, n.2, p. 362-384, 2013.

BEICHNER, J. R. American Journal of Physics. N.62, p. 750 762, 1994.

BEICHNER, J. R. American Journal of Physics. N.64, 1996.

BEZERRA JUNIOR, A. G.; LENZ, J. A.; OLIVEIRA, L. P.; SAAVEDRA, N. Manual para Usuários Iniciantes no software Tracker. i f., Universidade Tecnológica Federal do Paraná. Curitiba, 2011.

BROWN, D. COX, A. J. The Physics Teacher, v.47, p. 145-150, 2009.

CROUCH, C. H., MAZUR, E. American Journal of Physics. v. 69, n. 9, p. 970, 2001.

DA SILVA, J.C.G. Uma proposta de ensino de gráficos de cinemática com uso de videoanálise mediado por uma metodologia de aprendizagem ativa. 112f. Dissertação (Pós-graduação em Ciência Tecnologia e Educação) CEFET/RJ. Rio de Janeiro, Rio de Janeiro. 2016.

DE JESUS, Vitor L. B. Experimentos e videoanálise: Dinâmica. São Paulo: Editora Livraria da Física, 2014. 236p.

HAKE, R. American Journal of Physics. v. 66, p. 64- p.71. 1998.

MAZUR, E.; SOMERS, M. D. Upper Saddle River, N.J. Prentice Hall, 1997. 253 p.

MAZUR, E. Peer Instruction: A revolução da aprendizagem ativa / Eric Mazur; tradução: Anatólio Laschuk. Porto Alegre: Penso, p.252, 2015.

MORETTIN, P. A. e BUSSAB, W. de O. Estatística Básica. 6.ed. São Paulo: Saraiva, 2010.

MÜLLER, M. G.; BRANDÃO, R. V.; ARAUJO, I. S.; VEIT, E. A. Caderno Brasileiro de Ensino de Física, v. 29, p. 491-524, 2012.

MÜLLER, M. G., ARAUJO, I. S., VEIT, E. A., SCHELL, J. Revista Brasileira de Ensino de Física, Brasil, v. 39, n. 3, fev. 2017.v 
Revista do Professor de Física, v. 3, n. 2, p. 50-64, Brasília, 2019.

ZAVALA, G.; TEJEDA, S.; BARNOIL, P.; BEICHNER, R. J. Physical review physics education research, v. 13, 2017. 